\title{
Dialysis membranes: A 2018 update
}

\author{
Piotr Olczyk ${ }^{1, A-D}$, Artur Małyszczak ${ }^{1, A-D}$, Mariusz Kusztal ${ }^{2, E, F}$ \\ ${ }^{1}$ Faculty of Medicine, Wroclaw Medical University, Poland \\ ${ }^{2}$ Department of Nephrology and Transplantation Medicine, Wroclaw Medical University, Poland \\ A - research concept and design; $B$ - collection and/or assembly of data; $C$ - data analysis and interpretation; \\ $\mathrm{D}$ - writing the article; $\mathrm{E}$ - critical revision of the article; $\mathrm{F}$ - final approval of the article
}

Address for correspondence

Piotr Olczyk

E-mail: ol.piotr1994@gmail.com

Funding sources

None declared

Conflict of interest

None declared

Received on 0ctober 8, 2018

Reviewed on January 12, 2019

Accepted on January 16, 2019

Cite as

Olczyk P, Małyszzzak A, Kusztal M. Dialysis membranes:

A 2018 update. Polim Med. 2018;48(1):57-63.

doi:10.17219/pim/102974

DOI

10.17219/pim/102974

Copyright

@ 2019 by Wroclaw Medical University

This is an article distributed under the terms of the

Creative Commons Attribution Non-Commercial License

(http://creativecommons.org/licenses/by-nc-nd/4.0/)

\begin{abstract}
Dialysis membranes are the basic element of a hemodialyzer. Synthetic and natural materials characterized by various fiber arrangements are used in their production. The most up-to-date ones are made of synthetic polymers such as polyamide, phosphatidylserine (PS), polyacrylonitrile-based fiber (PAN), polyarylethersulfone, polyethersulfone, or polymethylmethacrylate. Dialysis membranes are characterized by the ability to remove uremic molecules, which can be divided into small water-soluble compounds, protein-bound compounds and larger "middle molecules". Newer membranes such as medium cut off membranes (MCO) allow the removal of a wider spectrum of uremic molecules, which reduces the risk of late complications of dialysis. Dialysis membranes are used in therapy methods such as low flux, high flux or HDx therapy. An important aim in dialysis membrane development is to increase their biocompatibility. Insufficient biocompatibility can result in complement activation or platelet activation, which can lead to an increased risk of cardiovascular complications. The aim of the study is to discuss the latest reports on dialysis membranes.
\end{abstract}

Key words: dialysis, membranes, HDx, MCO 
Despite the increasing frequency of kidney transplants, the number of patients requiring hemodialysis (HD) continues to grow. According to the European Renal Association registry on December 31, 2014, 490,743 individuals in Europe were receiving renal replacement therapy for end-stage renal disease (ESRD), equating to an unadjusted prevalence of 924 patients per million population (pmp) (ranging from 157 pmp in Ukraine to $1794 \mathrm{pmp}$ in Portugal). In 2016, 20,144 Polish patients required HD, ${ }^{1}$ and were struggling with numerous complications caused by insufficient removal of uremic molecules.

It is worth mentioning the classifications of the European Uremic Toxin Work Group (EUTox) here: 1. small water-soluble compounds; 2 . protein-bound compounds; and 3. larger "middle molecules". High-flux dialysis and more efficient treatment techniques, like hemodiafiltration (HDF), improve the removal of uremic toxins in the middle molecular-weight range.

Currently, the most popular (and low cost) dialysis membranes are synthetic membranes, used in high-flux dialysis. In recent years, a new type of membrane - medium cut-off membranes (MCO), used in expanded HD therapy (HDx) - has been developed. Today, new MCO membranes with increased pore size allow for the removal of toxins with bigger molecular weight, such as kappa and lambda light chains and/or mediators of inflammation. Heparin-grafted membranes have been developed to reduce the risk of bleeding. Graphene membranes are at the early phase of research. This article presents an overview of the dialysis membranes currently available and those which will soon appear on the market.

\section{Chemical composition and its influence on membrane performance}

An important aspect of the functioning of dialysis membranes is their composition. Formerly, they were divided into cellulose and noncellulosic. Nowadays, we distinguish rayon-based membranes (also known as cuprophan membranes) and other membranes such as cellulose acetate, cellulose triacetate and so on, also known as modified cellulosic membranes. The second group includes synthetic membranes made of polyamide, phosphatidylserine (PS), polyacrylonitrile-based (PAN) fibers, polyarylethersulfone, polyethersulfone, and polymethylmethacrylate. The membranes in the second group are characterized by better biocompatibility and the ability to remove substances of a bigger molecular weight. In order to reduce the side effects associated with excessive hydrophobicity and to increase the efficiency and permeability of the membrane, is possible to use compositions of different synthetic components. ${ }^{2,3}$ Excessive hydrophobicity is associated with membrane fouling, caused by adhe- sion of plasma proteins to the surface of the membrane. It leads to platelet adhesion, aggregation and coagulation.

Cellulose membranes are considered natural. They have a symmetrical structure and the same pore size in all layers. They also have hydrophilic properties, and after contact with blood and dialysate they take the form of a homogeneous gel. Some synthetic membranes have similar construction. An asymmetric structure is characteristic only of synthetic membranes. They have a thin, selective inner layer and a thicker outer support layer. The outer layer is formed of a finely porous skin, which is the real separation barrier for solute. The inner part of the support layer is characterized by its high density, which reduces from the inside to the outside. The support layer provides mechanical stability. Under the microscope it forms a sponge-like or finger-type structure. Synthetic membranes, unlike cellulose membranes, have a strongly hydrophobic component. Their structure depends on the rate of precipitation of the polymer in the presence of a non-solvent solution. . $^{4}$

Synthetic and cellulose membranes differ also in terms of the fiber arrangement. Cellulose membranes naturally have a wave-like structure, while synthetic fibers are crimped to produce a ripple pattern for better blood and dialysate distribution. This design prevents contact or excess packing among fibers, and thus allows for better matching of blood and dialysate flows across all the sections of the fiber bundle. ${ }^{6,7}$

A clinically important parameter is molecular weight cut-off. This coefficient describes the largest molecule that can pass through the membrane. For older types of membrane, this value is about 3,000 $\mathrm{Da}$, while for newer ones it is around 15,000 Da. In super high-flux dialysis, it can be as high as $65,000 \mathrm{Da}$. Effective removal of medium and large molecules is made possible through the use of a more uniform pore size and by increasing the average pore size. An additional benefit is the sharper cut-off in the sieving coefficient, which facilitates the passage of low molecular-weight proteins and decreases albumin loss. ${ }^{8,9}$

\section{The most popular materials used in dialysis membranes}

Polysulfone-based membranes (PSf) are characterized by good mechanical strength and high resistance to chemicals and to temperature. The most important advantages that make PSf useful in HD are:

- high biocompatibility;

- high permeability for low molecular-weight proteins;

- high retention of endotoxins;

- the possibility of trouble-free sterilization..$^{10}$

Such membranes also have some disadvantages. They can cause protein accumulation on the surface of the membrane, which results in reduced flow and changes in membrane selectivity. Additionally, the accumulated protein may cause activation of the immune system. ${ }^{11}$ 
Polyethersulfone (PES) is one of the most common materials used in the production of dialysis membranes. It is characterized by high oxidative, chemical and thermal resistance and has appropriate mechanical properties. Polyethersulfone does not change after sterilization. One of the most important features of PES as a material for HD membranes is its high permeability for low-molecular weight proteins. The main problem is the hydrophobic nature of this material, which contributes to membrane fouling. Nowadays, hydrophilic polymers are added to PES to minimize this phenomenon. The most popular combination is PES with polyvinylpyrrolidone (PVP). ${ }^{12}$

Cellulose triacetate (CTA) is a compound formed by replacing the hydroxyl groups of cellulose with a carboxylic group. It is characterized by high diffusion efficiency and structural homogeneity. ${ }^{13}$ Cellulose triacetate and synthetic membranes have similar permeability for small molecular uremic toxins, $\beta 2$-microglobulin $(\beta 2 \mathrm{M})$ and small molecules associated with proteins. Another important property of CTA is its low thrombogenetic potential. ${ }^{14}$

The asymmetric triacetate membrane (ATA), which was created by modifying CTA, is a new compound developed by the Nipro Corporation (Osaka, Japan). Its main properties are its asymmetrical structure and smooth surface, resulting in high permeability during massive filtration, less variation in permeation time, better biocompatibility, and lower protein adsorption. ${ }^{15}$

Polymethyl methacrylate (PMMA) gives membranes high biocompatibility and high permeability. The PMMA-based membranes have the ability to remove protein through permeation and absorption. This allows the removal of highweight molecules like the free light chain of immunoglobulins $(56,000 \mathrm{Da})$. Polymethyl methacrylate can be used to reduce inflammation in patients during $\mathrm{HD}$ by removing cationic compounds and cytokines. It also contributes to the preservation of muscle mass in the elderly (probably due to a decreased loss of amino acids) and a reduction in pruritus. ${ }^{16}$

Polyester polymer alloy (PEPA) is a polymer based on polyarylate and PES. It is comprised of 3 layers: an inner skin layer, a porous layer and an outer skin layer. An important feature of PEPA is its ability to filter endotoxins through the outer layer. The addition of PVP allows increased $\beta 2 \mathrm{M}$ excretion and reduced albumin loss. ${ }^{17}$

The creation of ethylene vinyl alcohol co-polymer (EVOH) is another attempt to reduce HD-related complications. The EVOH-based membranes are characterized by reduced neutrophil activation and thus a reduction in oxidative stress. The activation of platelets and generation of reactive oxygen species by activated neutrophils on the surface of EVOHbased membranes is lower than on PSf membranes; EVOH is associated with lower inflammation rates and reduced atherogenesis progression, which results in better peripheral blood circulation in patients. Due to their hydrophilic structure EVOH-based membranes do not require the addition of PVP. The EVOH can therefore effectively reduce the risk of cardiovascular $(\mathrm{Cv})$ events in HD patients. ${ }^{18}$
Polyacrylonitrile-based membranes were designed for patients with HD-related complications such as peripheral arterial disease or poor nutritional status. ${ }^{19}$ They are hydrophilic membranes characterized by high permeability and high specificity for low and medium molecular proteins. ${ }^{20}$

Heparin-grafted membranes were created for patients with a higher risk of bleeding. Classic anticoagulation therapy using unfractionated or fractionated heparin is associated with a risk of bleeding, hypoaldosteronism, dyslipidemia, hyperkalemia, pruritus, and osteoporosis. Heparin-grafted membranes can provide an alternative for these patients; research indicated that they offer results no worse than classic anticoagulation therapy. ${ }^{21}$ There are other methods of heparin-free HD, such as albumin priming, regional citrate anticoagulation or airless tubing. A combination of heparin-sparing methods is probably more effective than any single one. However, hard data to prove this hypothesis have not been published so far. ${ }^{22}$

The Massachusetts Institute of Technology (MIT; Cambridge, USA) is currently conducting research on graphene dialysis membranes. At the early stage of testing, these membranes are reported to offer up to 10 times higher filtration speeds than membranes currently in use. Modern dialysis membranes work fairly slowly due to their thickness, but this new graphene membrane can accelerate this process due to being less than 1 nanometer thick. ${ }^{23}$

\section{Solute removal}

During dialysis 3 processes allow solute removal: absorption, diffusion and convection. There are 3 main groups of uremic solutes: 1 . small molecules $(<500 \mathrm{Da})$, which are removed during the diffusion process; 2 . middle and large molecules (50-15,000 Da), removed by high flux dialysis; and 3. molecules bound with proteins weighing $500 \mathrm{Da}$, which are hard to remove and the dissociation process is time-consuming. ${ }^{24}$

Protein-bound uremic toxins (PBUTs) are nondialyzable using currently available membranes. Toxins like indoxyl sulfate (IS, $251 \mathrm{kD})$ and $p$-cresyl sulfate $(108 \mathrm{kD})$ possess a very high protein-binding capacity of $90 \%$ with albumin. The molecular complexes formed by the protein-bound aggregate are too large to pass through dialysis membranes, resulting in a low reduction rate of $35 \%$ per session regardless of membrane class. Continued systemic accumulation of these uremic toxins after HD is the main factor contributing to HD-induced cardiovascular syndrome. Due to the nondializability of PBUTs, other strategies reducing the generation or absorption of PBUTs (like pre- and probiotics) have been tried. P-cresol is a product of protein metabolism by gut bacteria; the administration of probiotics could decrease its production. ${ }^{25}$ Some modified therapies have been more effective than conventional HD at removing certain PBUTs: 
1. daily HD (compared with the standard 3 times/week scheme) lowered levels of glycation-related substances and advanced glycation end products (AGEs); 2. superflux cellulose triacetate membranes were superior to lowflux membranes for clearing IS; 3 . increasing the dialyzer mass transfer area coefficient and dialysate flow improved the removal of PBUTs; and 4. the addition of activated charcoal to the dialysate resulted in greater efficiency in removing PBUTs. ${ }^{26}$

Table 1. Classification of solutes based on molecular weight (adapted from Azar and Canaud ${ }^{48}$ )

\begin{tabular}{|c|c|}
\hline $\begin{array}{l}\text { Molecular weight } \\
\text { range [Da] }\end{array}$ & Classification of solutes \\
\hline$<500$ & $\begin{array}{l}\text { small molecules } \\
\text { urea, creatinine, phosphate }\end{array}$ \\
\hline $500-15,000$ & $\begin{array}{l}\text { middle molecules } \\
\text { vitamin } B_{12} \text {, vancomycin, insulin, endotoxin } \\
\text { fragments, parathormone, } \beta 2 \text {-microgobulin }\end{array}$ \\
\hline$>15,000$ & $\begin{array}{l}\text { large molecules } \\
\text { myoglobin, retinol-binding protein (RBP), } \\
\text { erythropoietin (EPO), albumin, transferrin }\end{array}$ \\
\hline
\end{tabular}

\section{Dialyzer characteristics}

The mass-transfer area coefficient (KoA) describes the amount of solute clearance through diffusion. It is determined for each substance of a particular dialyzer. In clinical practice, the KoA for urea is used and is provided by the manufacturer. The KoA is the mass transfer coefficient and $\mathrm{A}$ is the surface of the membrane.

Diffusion is the movement of solutes across the membrane caused by differences in the concentration gradient. The diffusion rate depends on the characteristics of the solute (protein binding, charge, size), the concentration gradient of the solute between the dialysate and the blood, as well the dialysis membrane surface area, porosity, type, and thickness.

Convection is the movement of solutes out of the blood compartment through the dialysis membrane, with the fluid being removed during ultrafiltration. Convective transport is independent of solute concentration gradients across the membrane. The size of the membrane pores determines which solutes can be removed.

During ultrafiltration fluid flows through the dialysis membrane. This movement is forced by a difference in pressure on the 2 sides of the membrane (the pressure gradient). The ultrafiltration coefficient (KUF), which is ultrafiltration rate (QUF) / pressure gradient in the membrane (deltaP), describes the efficiency of the membrane for ultrafiltration. ${ }^{27}$

Absorption takes place when molecules permeate the sorbent and are subsequently taken up by it. In addition, whereas some sorbents take up molecules until they become saturated, others act primarily by exchanging one molecule for another. This phenomenon can be used to remove a specific toxin from blood. Absorption is not used in conventional HD therapy, but work is currently underway to apply it in portable and wearable dialysis devices. $^{28}$

One of the most important parameters describing a dialyzer is clearance. Urea clearance can be used to calculate the dialysis dose. In patients with elevated levels of uric acid or phosphate, uric acid clearance and phosphate clearance can be useful, but these values are not always reported.

The introduction of dialyzers with enlarged pores has resulted in increases in middle molecule clearance, leading to a need to find a middle molecule marker. $\beta 2$-microglobulin $(\beta 2 \mathrm{M})$ is now used as a middle molecule marker, because it is easy to measure. High-flux dialyzers are characterized by a KUF coefficient $>15 \mathrm{~mL} / \mathrm{h} / \mathrm{mm} \mathrm{Hg}$ and an ability to clear $\beta 2 \mathrm{Ms}$ at a rate of more than $20 \mathrm{~mL} / \mathrm{min} .^{29}$ $\beta 2$-microglobulin clearance is also the basis for the 5 -level dialyzer classification system developed in Japan (Table 2). Grades 4 and 5 are characterized by clearance $\geq 70 \mathrm{~mL} / \mathrm{min}$ and a blood flow of $200 \mathrm{~mL} / \mathrm{min}$ with a dialysate flow of $500 \mathrm{~mL} / \mathrm{min}$. Dialysis membranes used in grade 4 and 5 dialyzers are called high-performance membranes (HPMs) due to their high flux rate, permeability and biocompatibility.

High-performance membranes have larger pores than low-performance membranes, which allows the removal of not only small molecules, but also medium and large molecules, including low-molecular weight proteins (LMWPs) and small amounts of albumin. The optimal pore size should prevent a loss of albumin $>3 \mathrm{~g} /$ session with a blood flow rate of $200 \mathrm{~mL} / \mathrm{min}$ and a dialysate flow rate of $500 \mathrm{~mL} / \mathrm{min} .{ }^{30}$ This structure makes the filtering of uremic toxins and albumin in the dialyzer similar to the human kidney. ${ }^{31,32}$

Table 2. Japanese dialyzer classifications

\begin{tabular}{|c|c|}
\hline Dialyzer class & Clearance of $\beta 2$-microglobulin of less than $[\mathrm{mL} / \mathrm{min}]$ \\
II & 10 \\
III & 30 \\
IV & 50 \\
V & 70 \\
\hline
\end{tabular}

\section{High-flux vs low-flux dialysis}

According to a review by Palmer et al., high-flux dialysis can reduce the mortality of hemodialyzed patients due to CV events by $15 \%$, but all-causes mortality was not significantly decreased. ${ }^{33}$ Another study showed that after 3.7 years of HD, the risk of cerebrovascular disease and cardiac mortality is significantly lower in the case of highflux dialysis than in low-flux dialysis. ${ }^{34}$ Despite its many advantages, high-flux dialysis can be associated with backflow, which rarely occurs with low-flux dialysis. This can be a problem when dialysis water is contaminated 
with endotoxins, because wash-out from the membrane can reach the blood side. However, nowadays it is not a common problem. In addition, high-flux dialysis may be associated with an increased risk of hypotension, especially in patients with impaired cardiac function or autonomic neuropathy. ${ }^{30}$

\section{Biocompatibility}

High biocompatibility is crucial for the long-term survival of dialysis patients. The contact of blood with artificial material (the dialysis membrane) may cause many complications. Adsorption of plasma proteins on the membrane surface impairs the functioning of the membrane and can trigger the activation of almost any plasma or cellular component of the blood. ${ }^{35}$ In the past, cuprophan membranes were used, but they were highly immunoreactive because of the large number of hydroxyl groups, responsible for complement activation and leukopenia. Technological advances made it possible to create synthetic membranes with reduced immunoreactivity. The following crucial issues are related to biocompatibility: complement activation, platelet activation and toxins.

Complement activation: the contact of plasma with the dialysis membrane can result in complement activation, which can lead to a pseudo-anaphylactic reaction called complement activation-related pseudoallergy (CARPA). ${ }^{36}$ It has been shown that complement activation is related to the progression of cardiovascular disease (CVD). According to several studies, patients with higher C3 levels are more likely to develop CV events. Poppelaars et al. reported that patients who develop CV events have sharp C3d/C3 ratio increases after 30 min of HD. ${ }^{37}$ Complement activation caused by the contact of blood with the dialysis membrane can lead to a higher frequency of thromboembolic events. ${ }^{38}$

Platelet activation: fibrinogen accumulates on the dialysis membrane, which results in the adhesion and activation of platelets. A decrease in the platelet count is usually observed for 15-30 min after HD and ranges from 5-15\%. Studies have shown that significant thrombocytopenia (defined a priori as a post-dialysis platelet count of less than $100 \times 10^{3} / \mu \mathrm{L}$ or a post-dialysis decrease in platelet count of more than $15 \%$ ) was most frequently observed in patients dialyzed with PSf membranes that were previously sterilized using an electron beam. ${ }^{39}$

Toxins: bisphenol A (BPA) is a component of some dialysis membranes. During HD, it is released and absorbed into the blood. In a patient with impaired kidney function, it accumulates. It can cause atherosclerosis, diabetes, metabolic syndrome, insulin resistance, obesity, and CVD. Ethylene oxide (ETO) is a sterilizing substance that can accumulate in dialyzers during contact with dialyzer components made of polycarbonates. The penetration of ETO into the blood can cause anaphylactic reactions.
Insufficient biocompatibility can lead to such clinical consequences as: pulmonary changes, worsening of renal function and dialysis-related amyloidosis.

Pulmonary changes: it has been suggested that less biocompatible membranes activate neutrophils and the complement system to a greater extent than newer, more biocompatible ones. The first reaction may result in the release of inflammatory mediators and reactive oxygen species (ROS) that could trigger a breakdown of pulmonary elastin fibrils, increasing the probability of developing emphysema. Increasing leukocyte sequestration in the pulmonary capillary network can lead to a serious diffusion defect. Enhanced complement activation (especially component $\mathrm{C5}$ ) has been linked to leukotriene release, smooth muscle contraction, pulmonary hypertension, and hypoxemia. Complement $\mathrm{C} 5 \mathrm{a}$ is an anaphylotoxin, so it promotes increases in vascular permeability and the progression of pulmonary edema. Studies have suggested that high biocompatibility plays an important role in preventing pulmonary dysfunction in HD patients, but there are also studies with contradictory results. ${ }^{40}$

Worsening of renal function: biocompatibility plays an important role in preserving residual renal function in HD patients. Factors like neutrophil and complement activation triggered by less biocompatible membranes can lead to a worsening of renal function. Both result in the release of vasoconstrictive compounds, which promotes ischemia, especially in the medullar region of the kidney. ${ }^{41}$

Dialysis-related amyloidosis: $\beta 2 \mathrm{M}$ is a protein present in almost all nucleated cells, and is additionally released by degranulating neutrophils. Its aggregation as amyloid fibrils can lead to dialysis-related amyloidosis, a well-known pathologic process in long-term HD patients. The symptoms include pathologic fractures, arthropathy and carpal tunnel syndrome. Less biocompatible membranes, such as cellulose membranes, are known to induce this process more rapidly than PAN or PMMA membranes. The mechanisms leading to this difference include: increased release of proteases and ROS from neutrophils, which enhance the aggregation of $\beta 2 \mathrm{M}$ into amyloid fibrils, increased synthesis of $\beta 2 \mathrm{M}$ by mononuclear cells and the fact that cellulosic membranes do not sufficiently clear $\beta 2 \mathrm{Ms} .{ }^{42}$

\section{New types of dialysis membrane and a new type of therapy}

One of the most important challenges of dialysis therapy is to reduce the number of late HD complications. Many side effects are associated with insufficient removal of middle and large molecules. Table 3 presents some of the correlations between clinical problems and uremiaretention molecules. The introduction of high-flux dialysis and HDF promised to make it possible to remove these molecules. Unfortunately, the effectiveness of these solutions remains unsatisfactory. ${ }^{43}$ 
Table 3. Associations between uremic-retention molecules and clinical problems (based on Lorenzin et al. ${ }^{45}$ )

\begin{tabular}{|c|c|}
\hline Uremic-retention molecule & Clinical problem \\
\hline$\beta 2$-microglobulin & dialysis-related amyloidosis \\
\hline $\begin{array}{l}\text { inflammatory mediators, leptin, } \\
\text { appetite-suppressing toxins }\end{array}$ & malnutrition \\
\hline $\begin{array}{l}\text { parathyroid hormone, homocysteine, } \\
\text { inflammatory cytokines }\end{array}$ & $\begin{array}{c}\text { cardiovascular complications } \\
\text { and osteodystrophy }\end{array}$ \\
\hline erythropoiesis inhibitors, hepcidin & anemia \\
\hline $\begin{array}{l}\text { polyclonal free light chains } \\
(\mathrm{k}-\mathrm{FLC} \text { and } \lambda-\mathrm{FLC} \text { ) }\end{array}$ & atherosclerosis \\
\hline
\end{tabular}

Medium cut-off membranes are a new solution that has appeared on the market. They are characterized by a narrow molecular weight interval between cut-off and retention onset (a sieving coefficient between 0.1 and 0.9). This has been achieved by tight pore size distribution, and it allows effective removal of middle-to-high-weight molecules with little albumin loss. ${ }^{44}$

In order to utilize the potential of $\mathrm{MCO}$ membranes, HDx was developed. This new therapy allows increased clearance $(\mathrm{K})$ of large molecules. However, due to the low diffusion coefficient of MCO membranes, they additionally require convection. This can be achieved in 2 ways: by increasing ultrafiltration (QUF) or by increasing sieving (S), since $\mathrm{K}=\mathrm{QUF} * \mathrm{~S}$. In HDF, QUF is increased. As a result, in $\mathrm{HDF}$, there is a need to replenish a large amount of commercially prepared fluids and to use special devices for fluid preparation. In contrast, in $\mathrm{HDx}$, it is possible to maintain a lower QUF by increasing $S$. This technique can be performed using standard equipment for HD. Specially prepared fluids are not required due to properly balanced reverse filtration. ${ }^{45,46}$

High cut-off (HCO) membranes are characterized by an increased average pore diameter and effective removal of substances in the range from $20 \mathrm{kDa}$ to $50 \mathrm{kDa}$. This modification allows the passage of larger proteins and more effective clearance of middle-molecular weight molecules, which permits the removal of excess myoglobin in trauma patients, inflammatory molecules in sepsis or free light-chain proteins in multiple myeloma. The downside of this approach is albumin loss, which limits its use in chronic HD. ${ }^{47}$

\section{Summary}

Despite the continuous development of technology and the emergence of more and more refined dialysis membranes, no membrane functions closely enough to the human kidney. Neither ideal biocompatibility nor the removal of the full spectrum of uremic molecules has been achieved. Removing middle molecules is possible through high-flux therapy and HDF. However, the efficiency of this process is insufficient, especially for cardiotoxins among proteinbound toxins. Currently, the membranes that have the most promising properties are MCO membranes. Their use in HDx makes efficient removal of middle molecules possible without excessive ultrafiltration. We are awaiting randomized controlled trials and long-term results of MCO membrane use in the dialysis population.

\section{ORCID iDs}

Piotr Olczyk (D) https://orcid.org/0000-0002-9447-9459

Artur Małyszczak (D) https://orcid.org/0000-0003-0167-5600

Mariusz Kusztal (D) https://orcid.org/0000-0002-6502-0374

\section{References}

1. Dębska-Ślizień A, Rutkowsk B, Rutkowski G, Korejwo R, Gellert R. Stan terapii nerkozastępczej w Polsce - 2016. Nefrol Dial Pol. 2018;22:1-8.

2. Ronco C. Hemodiafiltration: Technical and clinical issues. Blood Purif. 2015;40(Suppl 1):2-11.

3. Clark WR, Hamburger RJ, Lysaght MJ. Effect of membrane structure and composition on performance and biocompatibility in hemodialysis. Kidney Int. 1999;56(6):2005-2015.

4. Ronco C, Crepaldi C, Brendolan A, et al. Evolution of synthetic membranes for blood purification: The case of the Polyflux family. Nephrol Dial Transplant. 2003;18(Suppl 7):10-20, discussion 55.

5. Zweigert C, Neubauer M, Storr M, et al. Progress in the development of membranes for kidney-replacement therapy. In: Drioli $\mathrm{E}$, Giorno L, eds. Comprehensive Membrane Science and Engineering. Oxford, UK: Elsevier; 2013:351-387.

6. Davenport A. Membrane designs and composition for hemodialysis, hemofiltration and hemodiafiltration: Past, present and future. Minerva Urol Nefrol. 2010;62(1):29-40.

7. Ronco C, Levin N, Brendolan A, et al. Flow distribution analysis by helical scanning in polysulfone hemodialyzers: Effects of fiber structure and design on flow patterns and solute clearances. Hemodial Int. 2006;10(4):380-388.

8. Sakai K, Matsuda M. Solute removal efficiency and biocompatibility of the high-performance membrane from engineering point of view: High-performance membrane dialyzers. Contrib Nephrol. 2011;173:11-22.

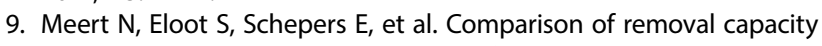
of two consecutive generations of high-flux dialysers during different treatment modalities. Nephrol Dial Transplant. 20011;26(8):2624-2630.

10. Su B-H, Shi Y, Fu P, Tao Y, Nie S, Zhao C-S. Clinical evaluation of polyethersulfone high-flux haemodialysis membrane compared to other membranes. J Appl Polym Sci. 2012;124(S1):91-98.

11. Wenten I, Aryanti P, Khoiruddin K, Hakim A, Himma N. Advances in polysulfone-based membranes for hemodialysis. JMSR. 2016;2(2):78-89.

12. Baihai S, Shudong S, Changsheng Z. Polyethersulfone Hollow Fiber Membranes for Hemodialysis. Progress in Hemodialysis - From Emergent Biotechnology to Clinical Practice. 2011. Doi:10.5772/22857.

13. Sunohara T, Masuda T. Cellulose triacetate as a high-performance membrane: High-performance membrane dialyzers. Contrib Nephrol. 2011;173:156-163.

14. Ronci M, Leporini L, Felaco P, et al. Proteomic characterization of a new asymmetric cellulose triacetate membrane for hemodialysis. Proteom Clin Appl. 2018;12(6):e1700140.

15. Sunohara T, Masuda, T. Fundamental characteristics of the newly developed ATA membrane dialyzer. Contrib Nephrol. 2017;189:215-221.

16. Aucella F, Vigilante M, Gesuete A. Review: The effect of polymethylmethacrylate dialysis membranes on uraemic pruritus. NDT Plus. 2010;3(Suppl 1):i8-i11.

17. Igoshi T, Tomisawa N, Hori Y, Jinbo Y. Polyester polymer alloy as a high-performance membrane. Contrib Nephrol. 2011;173:148-155.

18. Nakano A. Ethylene vinyl alcohol co-polymer as a high-performance membrane: An EVOH membrane with excellent biocompatibility. Contrib Nephrol. 2011;173:164-171.

19. Nakada H, Kashiwagi T, lino Y, Katayama Y. Therapeutic effects of the long-term use of PAN membrane dialyzer in hemodialysis patients: Efficacy in old dialysis patients with mild PAD. J Nippon Med Sch. 2014;81(4):221-235. 
20. Thomas M, Moriyama K, Ledebo I. AN69: Evolution of the world's first high permeability membrane. Contrib Nephrol.2011;173:119-129.

21. Meijers B, Metalidis C, Vanhove T, Poesen R, Kuypers D, Evenepoel $P$. A noninferiority trial comparing a heparin-grafted membrane plus citrate-containing dialysate versus regional citrate anticoagulation: Results of the CiTED study. Nephrol Dial Transplant. 2017;32(4):707-714.

22. Szymczak A, Kanafa M, Chuć M, Kusztal M. Hemodializa bez heparyny. Postepy Hig Med Dosw. 2018;72:671-677.

23. Chu J. Scientists produce dialysis membrane made from graphene. MIT News Office. http://news.mit.edu/2017/scientists-produce-dialysismembrane-made-from-graphene-0628. Accessed October 6, 2018.

24. Aucella F, Gesuete A, Vigilante M, et al. Adsorption dialysis: From physical principles to clinical applications. Blood Purif. 2013;35(Suppl 2):42-47.

25. Guo J, Lu L, Hua Y, et al. Vasculopathy in the setting of cardiorenal syndrome: Roles of protein-bound uremic toxins. Am J Physiol Heart Circ Physiol. 2017;1:313(1):1-13.

26. Meyer TW, Peattie JW, Miller JD, et al. Increasing the clearance of protein-bound solutes by addition of a sorbent to the dialysate. J Am Soc Nephrol. 2007;18(3):868-874.

27. Ficheux $A$, Ronco $C$, Brunet $P$, Argilés À. The ultrafiltration coefficient: This old 'grand inconnu' in dialysis. Nephrol Dial Transplant. 2015;30(2):204-208.

28. Davenport A. Portable and wearable dialysis devices for the treatment of patients with end-stage kidney failure: Wishful thinking or just over the horizon? Pediatr Nephrol. 2014;30(12):2053-2060.

29. Karkar A. Advances in hemodialysis techniques. Hemodialysis IntechOpen. http://www.intechopen.com/books/hemodialysis/advances-in-hemodialysis-techniques. Accessed October 6, 2018.

30. Saito A. Definition of high-performance membranes: From the clinical point of view. Contrib Nephrol. 2011;173:1-10.

31. Tsuchida K, Minakuchi J. Albumin loss under the use of high-performance membranes. Contrib Nephrol. 2011;173:76-83.

32. Niwa T. Update of uremic toxin research by mass spectrometry. Mass Spectrom Rev. 2011;30(3):510-521.

33. Palmer SC, Rabindranath KS, Craig JC, et al. High-flux versus lowflux membranes for end-stage kidney disease. Cochrane Database Syst Rev. 2012;12(9):CD005016.

34. Delmez JA, Yan G, Bailey J, et al; Hemodialysis (HEMO) Study Group. Cerebrovascular disease in maintenance hemodialysis patients: Results of the HEMO study. Am J Kidney Dis. 2006;47(1):131-138.

35. Pieroni L, Mortera SL, Greco V, et al. Biocompatibility assessment of haemodialysis membrane materials by proteomic investigations. Mol Biosyst. 2015;11(6):1633-1643.

36. Poppelaars F, Faria B, Costa MG, et al. The complement system in dialysis: A forgotten story? Front Immunol. 2018;9:71.

37. Poppelaars F, Gaya da Costa M, Faria B, et al. Intradialytic complement activation precedes the development of cardiovascular events in hemodialysis patients. Front Immunol. 2018;9:2070.

38. Lines SW, Richardson VR, Thomas B, Dunn EJ, Wright MJ, Carter AM Complement and cardiovascular disease: The missing link in haemodialysis patients? Nephron. 2016;134(2):103-103.

39. Kiaii M, Djurdjev O, Farah M, et al. Use of electron-beam sterilized hemodialysis membranes and risk of thrombocytopenia. JAMA. 2011;306(15):1679-1687.

40. Lang SM, Becker A, Fischer R, Huber RM, Schiffl H. Acute effects of hemodialysis on lung function in patients with end-stage renal disease. Wien Klin Wochenschr. 2006;118(3-4):108-113.

41. Hakim RM, Wingard RL, Parker RA. Effect of the dialysis membrane in the treatment of patients with acute renal failure. $N$ Engl J Med. 1994;331(20):1338-1342.

42. Hakim RM. Clinical implications of hemodialysis membrane biocompatibility. Kidney Int. 1993;44(3):484-494.

43. Wolley M, Jardine $M$, Hutchison CA. Exploring the clinical relevance of providing increased removal of large middle molecules. Clin $J$ Am Soc Nephrol. 2018;13(5):805-814.

44. Kirsch AH, Lyko R, Nilsson LG, et al. Performance of hemodialysis with novel medium cut-off dialyzers. Nephrol Dial Transplant. 2017;32(1):165-172.

45. Lorenzin A, Neri M, Lupi A, et al. Quantification of internal filtration in hollow fiber hemodialyzers with medium cut-off membrane. Blood Purif. 2018;46(3):196-204.
46. Ronco C, The rise of expanded hemodialysis. Blood Purif. 2017;44(2):I-VIII.

47. Ronco C, Manna GL. Expanded hemodialysis: A new therapy for a new class of membranes. Contrib Nephrol. 2017;190:124-133.

48. Azar T, ed. Modelling and Control of Dialysis Systems. Berlin, Germany: Springer-Verlag; 2013. 
